\title{
Extension des trématodoses du bétail après la construction des barrages dans le bassin du fleuve Sénégal
}

\author{
O.T. Diaw ${ }^{1}$ G. Vassiliades ${ }^{1}$ Y. Thiongane ${ }^{2}$ \\ M. Seye ${ }^{1}$ Y. Sarr ${ }^{1}$ A. Diouf ${ }^{1}$
}

Mots-clés

Bovin - Caprin - Ovin - Fasciola gigantica - Schistosoma bovis Paramphistomum sp. - Epidémiologie Barrage - Delta - Lac - Sénégal.

\begin{abstract}
Résumé
Après la mise en service du barrage de Diama (1985-86) et la multiplication des aménagements hydro-agricoles, il a été constaté au niveau du bassin du fleuve Sénégal un développement des trématodoses animales, particulièrement la fasciolose à Fasciola gigantica, les schistosomoses à Schistosoma bovis et à S. curassoni et la paramphistomose à Paramphistomum sp. Cette situation épidémiologique des trématodoses chez le bétail s'est manifestée par une augmentation des prévalences au niveau d'anciens foyers (Richard-Toll, Ross-Béthio, M bane et Keur Momar Sarr). Les taux d'infestation chez les bovins sont passés de 11 à 27 p. 100, de 20 à 30 p. 100 et de 15 à 27 p. 100 respectivement pour la fasciolose, la paramphistomose et la schistosomose. Chez les petits ruminants qui semblaient être épargnés, des prévalences de 2 à 62 p. 100 pour la fasciolose ont été enregistrées, alors que la paramphistomose, plus fréquente, avait un taux de 25 à 30 p. 100. Parallèlement, à partir de 1989-1990 de nouveaux foyers de trématodoses sont apparus : d'une part, au niveau du delta à Tilène, Pont Gendarme et Takhembeut avec des prévalences de 3 à 20 p. 100, 4 à 20 p. 100 et 5 à 36 p. 100 respectivement pour la fasciolose, la schistosomose et la paramphistomose ; d'autre part, au niveau du lac de Guiers à Temeye, Thiago et Senda avec des prévalences de 5 à 86 p. 100, 5 à 11 p. 100 et 5 à 33 p. 100 respectivement pour la fasciolose, la schistosomose et la paramphistomose. Au niveau de ces nouveaux foyers, des prévalences de 2 à $55 \mathrm{p}$. 100 pour la fasciolose et de 5 à $25 \mathrm{p}$. 100 pour la paramphistomose ont été enregistrées chez les petits ruminants. La schistosomose était moins fréquente avec des prévalences de 2 à 4 p. 100. Cette nouvelle situation épidémiologique de trématodoses dans le bassin du fleuve Sénégal à partir de 1988-1989 était surtout remarquable par les prévalences très élevées de ces affections, les fortes charges parasitaires et par un polyparasitisme associant douves, schistosomes et paramphistomes.
\end{abstract}

\section{INTRO DUCTION}

Dans le contexte évolutif lié aux modifications de l'environnement, la vallée du fleuve Sénégal a connu de grands bouleversements durant ces dix dernières années.

Après la série des années de sécheresse de 1972-74 et 1982-84, deux barrages ont été construits sur le cours du fleuve Sénégal : celui de Diama en 1986 et de Manantali en 1990. Le premier est un barrage anti-sel situé à $33 \mathrm{~km}$ en amont de Saint-Louis. Il permet d'arrêter la remontée de la langue salée qui arrivait jusqu'à $250 \mathrm{~km}$ en amont de Saint-Louis et de constituer une réserve d'eau douce pendant toute l'année. Le second, construit en 1989-90, renforce le premier et régularise le cours du fleuve.

1. Service de parasitologie, Lnerv/Isra, BP 2057, Dakar-Hann, Sénégal

2. Service de virologie, Lnerv/Isra, BP 2057, Dakar-Hann, Sénégal
Cette mise en service des barrages a permis le développement de l'irrigation et la multiplication des aménagements hydro-agricoles (de grands périmètres d'irrigation sont actuellement aménagés tandis que des périmètres anciens sont peu à peu remis en état).

Cette mise en fonction des barrages ainsi que les aménagements hydro-agricoles associés ont pour corollaire des perturbations écologiques telles que la création de lacs de retenue, de biefs, de canaux d'irrigation, de drains et de vastes surfaces permanentes d'eau douce. Tous ces points d'eau constituent des habitats favorables au développement des mollusques aquatiques hôtes intermédiaires de trématodes.

La mise en valeur de ressources hydrauliques et le développement des mollusques sont des conditions favorables bien connues pour ce qui concerne l'apparition de trématodoses humaines (bilharzioses), mais ces conditions ont été peu évaluées pour les trématodoses animales. Dans ce contexte de bouleversement après la mise en service des barrages (1988-1989), les auteurs ont étudié l'évolution et l'extension des trématodoses animales au niveau du bassin du fleuve Sénégal, dans le delta et le lac de Guiers. 
De 1972 à 1984, du fait de la sécheresse, les trématodoses animales en général et la fasciolose en particulier ont fortement régressé. Dans le delta, les prévalences sont passées de 58 à 12 p. 100, alors qu'au niveau du lac de Guiers la prévalence la plus élevée enregistrée dans les années 1980 était de 5 p. 100 (16, 17 et statistiques du Service régional de la santé animale de Saint-Louis).

Puis, à partir de 1988, après la construction du barrage de Diama il a été constaté de nombreux cas de trématodoses animales dans le delta et le lac de Guiers avec souvent de fortes prévalences allant de 35 à 50 p. 100 chez les bovins et les petits ruminants.

Cette situation épidémiologique n'a cessé de s'aggraver et de 1990 à 1994 de nombreux foyers de trématodoses (fasciolose et schistosomose) ont été enregistrés.

Les pathologies, notamment les bilharzioses urinaire et intestinale, consécutives aux bouleversements écologiques ont été bien étudiées pour ce qui relève de la santé humaine. Les problèmes sanitaires causés par les trématodoses concernent également la santé animale mais n'ont pas été suffisamment pris en compte dans l'épidémiologie parasitaire animale en Afrique.

L'objectif de cette étude était de déterminer la prévalence et l'intensité des principales trématodoses animales (fasciolose, schistosomose et paramphistomose) dans le bassin du fleuve Sénégal (delta et lac de Guiers) et de montrer leur extension favorisée par les modifications actuelles de l'environnement, particulièrement après la construction des barrages.

\section{ATERIEL ET METHODES}

\section{Le milieu}

A l'exception de la zone côtière Nord, l'ensemble du bassin du fleuve Sénégal est situé dans la zone sahélienne caractérisée par une longue saison sèche de novembre à juin et une pluviométrie particulièrement défavorable $(385 \mathrm{~mm}$ à Saint-Louis, $300 \mathrm{~mm}$ à Podor).

Le climat chaud et sec est soumis une grande partie de l'année à l'harmatan. La végétation est réduite à des arbustes peu nombreux, épineux pour la plupart. En hivernage, l'herbe pousse en tapis continu de graminées et constitue l'essentiel des pâturages pour le bétail.

La zone d'étude est constituée par le delta ou région du bas Sénégal (de Saint-Louis à Dagana) auquel est associé le lac de Guiers qui communiquait avec le lit principal du fleuve Sénégal au niveau de Richard-Toll par le marigot de la Touey. Le tracé du marigot a été rectifié et remplacé par un canal rectiligne de Richard-Toll au lac de Guiers assurant un meilleur remplissage de ce dernier.

Le cheptel est constitué de zébus Peuls sénégalais ou Gobras. L'élevage est très développé et plus intensif au niveau du lac de Guiers que du delta qui est surtout aménagé pour l'agriculture (irrigation pour le riz principalement).

Depuis l'avènement des barrages, cette région dispose d'eau douce toute l'année, tant au niveau du delta que du lac de Guiers où le niveau de l'eau varie peu. La disponibilité permanente d'eau douce permet de meilleurs remplissages annuels et une plus grande stabilité du niveau du lac. Ces nouvelles conditions ont entraîné l'adoucissement progressif des eaux du lac et une forte régression des variations annuelles de la salinité autrefois importantes (4).

\section{Les sites de l'étude}

Les sites de l'étude les plus anciens étaient localisés au niveau des localités de Richard-Toll, de Ross-Béthio (delta), de Mbane et de Keur Momar Sarr (lac de Guiers). C'étaient les principaux foyers de trématodoses animales connus avant la construction des barrages (figure 1).

Après les modifications de l'environnement liées aux barrages dans les années 1988-1990, de nouveaux foyers de fasciolose et de schistosomose animale sont apparus dans le delta à Tilène, Pont Gendarme et Takhembeut et dans le lac de Guiers à Thiago, Temeye et Senda.

\section{Logique de l'étude}

L'objectif était de montrer l'évolution et l'extension des trématodoses animales en relation avec les modifications du milieu consécutives à la construction des barrages qui ont entraîné une prolifération des mollusques hôtes intermédiaires de trématodes (7).

Ceci s'est traduit à partir de 1989, après la mise en service des barrages, par l'augmentation des prévalences et de l'intensité de ces affections au niveau des anciens foyers et par l'extension de ces trématodoses dans de nouveaux foyers.

\section{Enquêtes parasitologiques et malacologiques}

\section{Enquêtes parasitologiques au niveau des anciens foyers}

Des enquêtes parasitologiques ont été effectuées chez le bétail au niveau des anciens foyers de Richard-Toll, de Ross-Béthio, de Mbane et de Keur Momar Sarr. Elles ont permis de connaître la situation épidémiologique des trématodoses dans le bassin du fleuve Sénégal $(16,17)$. Les prévalences ont été établies par sondages coprologiques (8) et par étude des animaux abattus aux abattoirs.

L'échantillonnage en grappe a été choisi comme méthode de sondage. Des troupeaux ont été sélectionnés et des prélèvements de fèces ont été faits sur tous les animaux. La taille des troupeaux a varié de 10 à 100 animaux.

Au niveau des abattoirs de Saint-Louis et de Richard-Toll, les prévalences des principales trématodoses ont été établies en recherchant les trématodes localisés au niveau du foie (observation macroscopique des canaux pour la récolte de Fasciola gigantica et de Dicrocoelium hospes), de la panse (mise en évidence des paramphistomes) et des mésentères (mise en évidence des schistosomes). L'examen microscopique du parenchyme hépatique a révélé la présence d'œufs de Schistosoma bovis, de $S$. curassoni et de Dicrocoelium.

\section{Enquêtes parasitologiques au niveau des nouveaux foyers}

De 1988 à 1994 de nouveaux foyers ont été observés au niveau du delta (Tilène, Pont Gendarme et Takhembeut) et du lac de Guiers (Temeye, Thiago et Senda). Les enquêtes ont été effectuées au niveau de ces sites à la suite de nombreux cas de mortalité et/ou de morbidité chez les animaux. Lors de ces enquêtes tous les animaux présentant des troubles cliniques ont été observés et des prélèvements de fèces ont été faits pour des analyses coprologiques (8). Le nombre d'animaux a varié en fonction de la taille des troupeaux.

Certains animaux en phase terminale ont été sacrifiés, puis autopsiés, pour compléter le diagnostic (observation des lésions, prélèvements d'organes, récolte de parasites, etc.). 


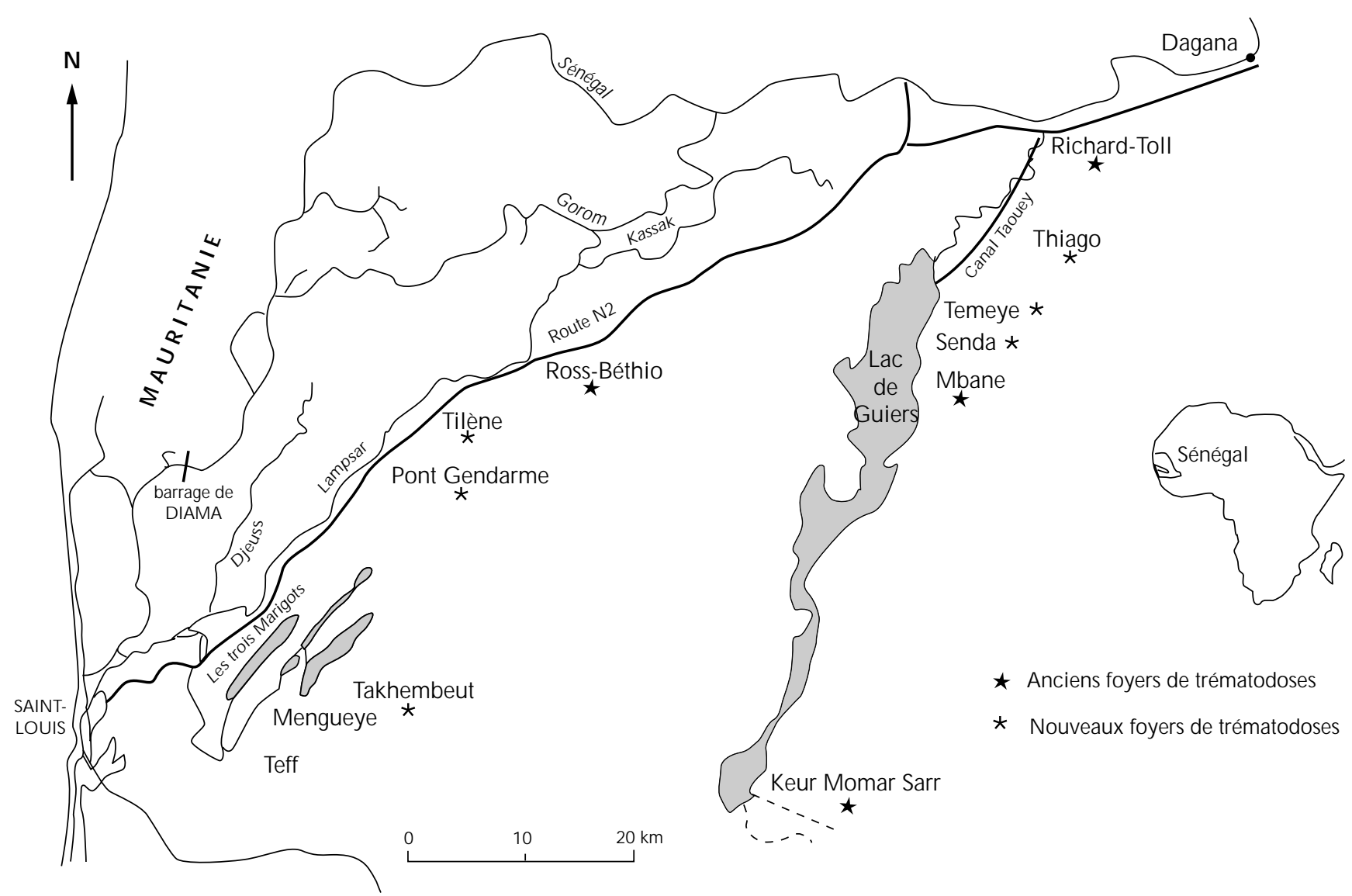

Figure 1 : delta du fleuve Sénégal et lac de Guiers - foyers de trématodoses du bétail.

\section{Enquêtes malacologiques}

Parallèlement aux enquêtes parasitologiques, des prospections malacologiques ponctuelles ont été effectuées au niveau de ces sites dans les zones de pâture et aux points d'abreuvement des animaux (mares, marigots, fleuve, lac, etc.).

Les mollusques ont été recherchés dans la végétation, dans la boue et au niveau des différents débris pouvant servir de supports. La recherche s'est effectuée à l'aide d'une épuisette munie d'un manche de 1,5 m de long. Les mollusques ont été récoltés et conservés dans des pots de prélèvement puis ramenés au laboratoire pour être examinés. Les différents mollusques ont été identifiés selon la clé de Mandahl Barth (12) et de Brown (3), puis groupés par espèce pour les dénombrer.

Afin d'étudier leur infestation, ces mollusques ont été mis dans des piluliers avec de l'eau distillée et exposés individuellement à la lumière du soleil ou celle d'une lampe pendant 10 à 15 min pour favoriser la sortie des cercaires. Ces dernières ont été récoltées et identifiées selon la clé de Frandsen et Christensen (9). Ces parasites peuvent aussi être déterminés par chétotaxie (13) et l'identification est confirmée par des infestations expérimentales de rongeurs (identification des adultes et des œufs après 30 à 45 jours).

Ainsi pour chaque espèce de mollusque récoltée on détermine la nature et le taux d'infestation parasitaire ce qui permet d'établir son rôle épidémiologique dans la transmission des trématodoses. La densité malacologique est exprimée par le nombre de mollusques récolté par une personne pendant une heure.

Ces enquêtes malacologiques, ponctuelles et transversales ont été effectuées au niveau des anciens foyers et des nouveaux sites.
RESU LTATS

\section{Situation épidémiologique dans les anciens foyers du delta et du lac de Guiers}

\section{Situation avant l'avènement des barrages}

En 1971, les enquêtes au niveau du delta et du lac de Guiers dans ces quatre principaux sites établissaient l'existence de la fasciolose dont la prévalence variait de 58 à 60 p. 100 chez les bovins. Les paramphistomoses avaient une prévalence de 34 p. 100 et la schistosomose, plus faible, de 5 p. 100 (16).

Un suivi aux abattoirs de Saint-Louis en février, mai et octobre 1978 ont permis de constater la diminution de la prévalence de la fasciolose avec respectivement des valeurs de 16, de 6 et de 7 p. 100 .

De 1974 à 1984, les données statistiques fournies par les agents des secteurs de l'élevage au niveau des abattoirs de Saint-Louis, de Richard-Toll et de Dagana ont permis d'établir la situation des trématodoses animales dans le delta et le lac de Guiers. Ainsi, a-t-il été rapporté en 1985 une forte baisse des principales trématodoses. Les niveaux moyens des prévalences étaient de 11 , de 15 et de 20 p. 100 respectivement pour la fasciolose, la schistosomose et la paramphistomose (statistiques du Service régional de la santé animale de Saint-Louis).

Chez les petits ruminants, les prévalences étaient presque nulles et quelques rares cas de paramphistomoses ont été signalés. 


\section{Situation après la mise en service des barrages}

Des sondages coprologiques effectués au niveau de Richard-Toll, de Ross-Béthio et de Mbane en janvier 1989 et octobre 1991 sur 150 bovins ont montré une forte évolution des prévalences des trématodoses (figure 2, tableau I).

A Mbane, il faut signaler en juillet 1988 une importante épidémie de fasciolose ovine, la première enregistrée au niveau du bassin du fleuve Sénégal, avec une prévalence de 62 p. 100 (15).

$\mathrm{Au}$ niveau des abattoirs de Richard-Toll et de Saint-Louis, entre 1989 et 1991, les agents d'élevage chargés des inspections ont enregistré une augmentation des cas de fasciolose ovine dont la prévalence variait de 2 à 11 p. 100. Les caprins étaient faiblement infestés (0,5 p. 100) (statistiques du Service régional de la santé animale de Saint-Louis).

En 1992, sur un total de 250 petits ruminants de la zone de RossBéthio, les analyses coprologiques ont révélé une prévalence de 15 p. 100 pour la fasciolose et de 25 p. 100 pour la paramphistomose.

Des prospections malacologiques au niveau des principaux points d'eau (points d'abreuvement) fréquentés par les animaux de ces sites ont montré une forte prolifération des mollusques surtout dans les marigots et le lac de Guiers. Des mollusques pulmonés, particulièrement les limnées qui colonisent un plus grand nombre de sites, pullulaient et une augmentation des bulins a également été enregistrée.

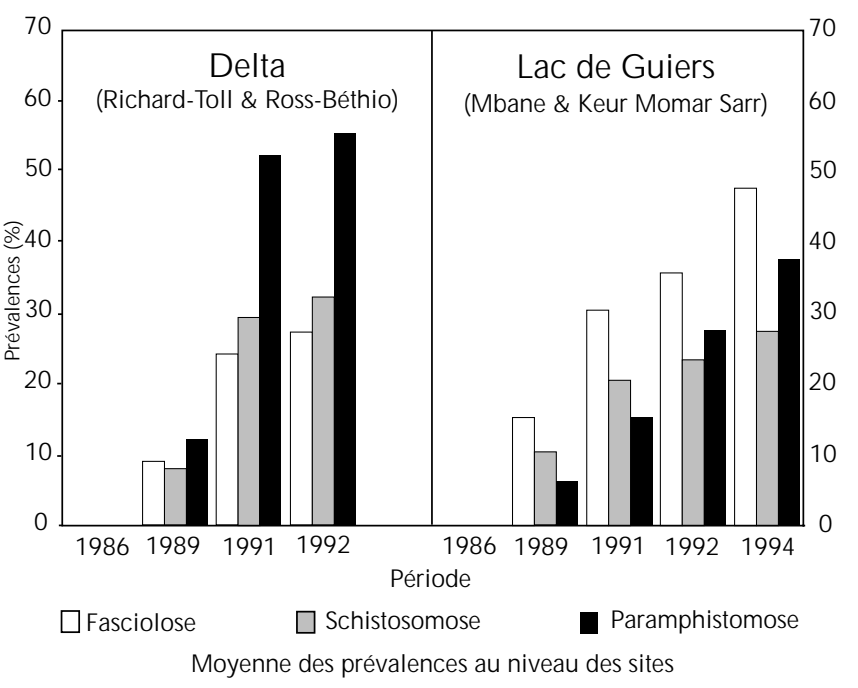

Figure 2 : évolution des trématodoses du bétail dans le bassin du fleuve Sénégal. Prévalences chez les bovins au niveau des anciens foyers (1988-1994).
Sur un total de 150 limnées récoltées dans trois sites, une infestation globale de 15 p. 100 a été relevée avec $F$. gigantica (1). De même des Bulinus truncatus (25 sur 100) étaient porteurs de cercaires de paramphistomes identifiées comme étant Paramphistomum phillerouxi $(2,13)$.

A Keur Momar Sarr, depuis 1990, de nombreux cas de fasciolose bovine ont été signalés. Ainsi une enquête en janvier 1992 portant sur six troupeaux de 30 à 80 bovins a révélé de fortes prévalences variant d'un élevage à l'autre : fasciolose 20 à 35 p. 100, schistosomose 15 à 23 p. 100 et paramphistomose 20 à 27 p. 100 . Chez les petits ruminants (100 sujets) des prévalences de fasciolose de 12 à 15 p. 100 et de paramphistomose de 25 à 30 p. 100 ont été enregistrées.

Cette situation n'a cessé d'évoluer et en 1994 un foyer de fasciolose est apparu. Une enquête a été effectuée en mars 1994 et a permis d'enregistrer une prévalence globale de 47 p. 100 de fasciolose sur les 350 animaux observés fréquentant le lac de Guiers. De plus, des cas de schistosomose (27 p. 100) et de paramphistomoses (37 p. 100) ont été observés.

Dès lors, les bergers connaissant les dangers du lac n'y conduisaient plus les petits ruminants, ces affections leur ayant ainsi été épargnées (aucun cas de trématodose sur les 150 analyses coprologiques).

Devant cette situation épidémiologique, une expérience a été tentée afin de montrer la permanence des risques de transmission de la fasciolose au niveau du lac et aussi la réceptivité des petits ruminants (introduction d'animaux traceurs). Ainsi, en septembre 1994 des « animaux de case » (trois chèvres et trois moutons) indemnes de trématodoses ont été introduits dans la zone du lac au niveau du village de Louboudou. Une fois marqués (boucle à l'oreille), ils ont été confiés à un berger du village et, comme les autres animaux de la zone, ont fréquenté le lac pour s'abreuver et ont utilisé les pâturages qui le bordaient. Après un séjour de 30 à 45 jours, une chèvre et un mouton sont morts atteints de fasciolose. A l'autopsie, de jeunes douves ont été récoltées dans le foie. Les autres animaux ont été transférés à Dakar dans une étable du service de parasitologie pour y être suivis. Après 75 jours, les quatre animaux restants ont été sacrifiés. A l'autopsie, un second mouton et une deuxième chèvre se sont révélés infestés. Des lésions au niveau du foie et la présence de douves dans les canaux hépatiques ont été observés. Sur les six animaux exposés, quatre ont été infestés (deux moutons et deux chèvres).

Cette étude expérimentale a montré que le lac de Guiers était une zone à haut risque de transmission de la fasciolose, ce qui explique les fortes prévalences de cette affection chez le bétail exposé.

Des bulins, des Biomphalaria et des limnées (Lymnaea natalensis) ont été récoltés, mais seules ces dernières ont révélé une infestation par Fasciola gigantica (1) avec un taux de 25 p. 100 (28/102).

\section{Tableau I}

Evolution des prévalences de trématodoses chez les bovins à Richard-Toll, Ross-Béthio et $M$ bane (enquêtes coprologiques en janv. 1989 et oct. 1991 sur 150 bovins)

\begin{tabular}{|c|c|c|c|c|c|c|}
\hline \multirow[b]{2}{*}{ Périodes et prévalences } & \multicolumn{2}{|c|}{$\%$ de fasciolose } & \multicolumn{2}{|c|}{ \% de schistosomose } & \multicolumn{2}{|c|}{$\%$ de paramphistomose } \\
\hline & Janv. 89 & 0 ct. 91 & Janv. 89 & 0 ct. 91 & Janv. 89 & 0 ct. 91 \\
\hline Richard-Toll & 10 & 15 & 8 & 33 & 15 & 43 \\
\hline Ross-Béthio & 8 & 32 & 8 & 25 & 8 & 60 \\
\hline Mbane & 15 & 30 & 10 & 20 & 6 & 15 \\
\hline
\end{tabular}


De 1988 à 1994, les données obtenues concernant les trématodoses du bétail au niveau des sites du delta (Richard-Toll et RossBéthio) et ceux du lac de Guiers (Mbane et Keur Momar Sarr) ont montré une progression des prévalences de la fasciolose, de la schistosomose et de la paramphistomose.

Concernant les petits ruminants, c'est à partir de 1988, juste après la construction du barrage de Diama, que les premiers cas de fasciolose et de schistosomose ont été enregistrés.

\section{Situation épidémiologique dans les nouveaux foyers de trématodoses}

C'était la première fois que des cas de trématodoses animales étaient signalés au niveau de Tilène, Pont Gendarme et Takhembeut dans le delta, et de Senda, Temeye et Thiago dans le lac de Guiers (figure 3). Jusqu'en 1985-1986 (avant la construction des barrages), les enquêtes antérieures n'avaient jamais reporté l'existence de ces foyers de trématodoses $(16,17)$.

\section{Situation dans les foyers du delta}

\section{- Foyers de Tilène et de Pont Gendarme}

En février 1990, des sondages coprologiques ont été effectués sur quatre des sept troupeaux de bovins qui existaient à Tilène. De même, des prélèvements de fèces ont été faits sur trois des cinq troupeaux de Pont Gendarme. La taille des troupeaux a varié de 10 à 30 animaux. Les analyses coprologiques ont révélé la présence de trématodes : Fasciola gigantica, Schistosoma bovis, S. curassoni et Paramphistomum sp. C'était la première fois que des trématodoses étaient signalées dans cette zone du delta. Les prévalences ont varié d'un troupeau à l'autre et d'un site à l'autre. A Tilène 80 bovins et 110 petits ruminants et à Pont Gendarme 65 bovins et 90 petits ruminants ont été observés. Les prévalences enregistrées au niveau des deux sites se sont présentées ainsi : fasciolose 3 à 20 p. 100, schistosomose 4 à 10 p. 100 et paramphistomose 5 à 36 p. 100.

Chez les petits ruminants ces mêmes affections ont été signalées, mais avec des prévalences plus faibles : la fasciolose a varié de 2 à 8 p. 100, la schistosomose de 2 à 4 p. 100 et la paramphistomose de 5 à 10 p. 100 (tableau II).

Tous les animaux de Tilène et de Pont Gendarme fréquentaient le marigot Lampsar.

La prospection malacologique effectuée au niveau du marigot Lampsar dans les zones d'abreuvement des animaux a permis de

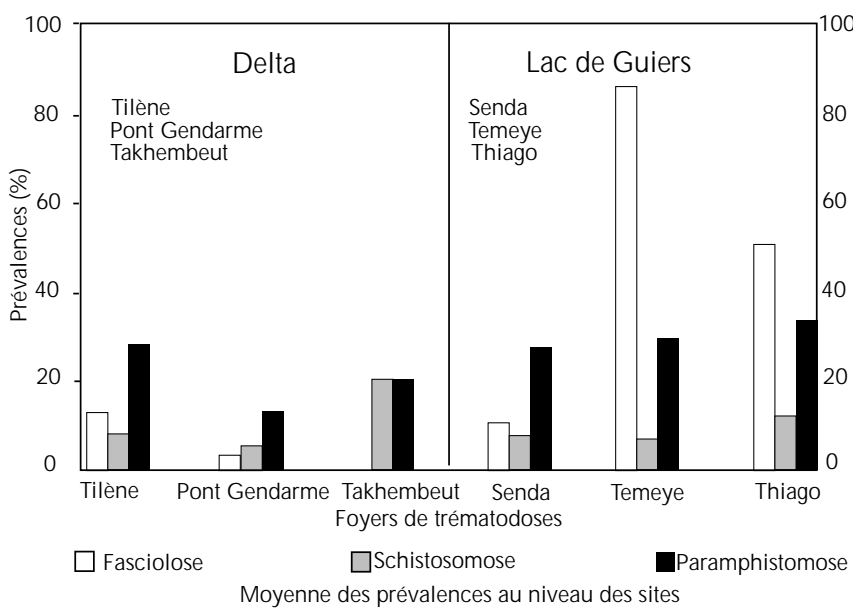

Figure 3 : nouveaux foyers de trématodoses dans le delta du fleuve Sénégal et le lac de Guiers. Prévalences de la fasciolose, de la schistosomose et de la paramphistomose chez les bovins (1990-1993).

récolter les mollusques suivants : Lymnaea natalensis (30 spécimens), Bulinus truncatus (50 spécimens), B. globosus (25 spécimens) et $B$. forskalii (15 spécimens). L'étude de ces mollusques au laboratoire a montré les taux d'infestation suivants : 50 p. 100 chez Lymnaea natalensis avec $F$. gigantica, 25 p. 100 chez $B$. truncatus avec $S$. bovis et 5 p. 100 chez B. globosus avec $S$. curassoni (9).

\section{- Foyer de Takhembeut}

En mai 1993, des cas de mortalité ont été enregistrés dans la zone des trois marigots à Takhembeut, Mengueye et Teff mais n'ont concerné que les bovins (104 sur 510, soit 20 p. 100 des animaux des troupeaux visités). Les petits ruminants ont été épargnés car ils ne fréquentaient pas cette zone de transhumance et utilisaient des pâturages éloignés des points d'eau.

Cinq troupeaux et campements d'éleveurs ont été visités ainsi que les points d'eau environnants qui font partie d'un ensemble constitué par des mares temporaires et par la zone des trois marigots.

Les études coprologiques sur 100 bovins ont montré une prévalence de 20 p. 100 de schistosomose à Schistosoma bovis et de 20 p. 100 de paramphistomose (tableau II). Les trématodoses étaient dominantes avec souvent des associations parasitaires. Dix pour cent d'animaux hébergeaient des schistosomes et des paramphistomes.

\section{Tableau II}

Prévalences des trémato doses au niveau des nouveaux foyers apparus à partir de 1990 chez des bovins et des petits ruminants (PR) du delta et du lac de Guiers

\begin{tabular}{|c|c|c|c|c|c|c|}
\hline \multirow[b]{2}{*}{ Foyers de trématodoses } & \multicolumn{2}{|c|}{$\%$ de fasciolose } & \multicolumn{2}{|c|}{ \% de schistosomose } & \multicolumn{2}{|c|}{$\%$ de paramphistomose } \\
\hline & Bovins & PR & Bovins & PR & Bovins & PR \\
\hline Tilène (1990) & 5 à 20 & 2 à 8 & 5 à 10 & 2 à 4 & 20 à 36 & 5 à $10 \%$ \\
\hline Pont Gendarme (1990) & 1 à 5 & & 1 à 8 & & 5 à 20 & \\
\hline Takhembeut (1993) & négatif & négatif & 20 & négatif & 20 & négatif \\
\hline Senda (1991-1992) & 5 à 15 & 20 à 50 & 4 à 10 & négatif & 24 à 30 & 15 à 25 \\
\hline Temeye (1992) & 86 & 28 & 6 & négatif & 29 & 15 \\
\hline Thiago (1992) & 50 & & 11 & & 33 & \\
\hline
\end{tabular}


L'autopsie d'un bovin malade sacrifié a révélé la présence de Schistosoma bovis avec de fortes charges parasitaires (200 schistosomes au niveau des mésentères et du foie) et une panse entièrement tapissée de paramphistomes, Paramphistomum phillerouxi (2) (confirmation par Dr Otto Sey, comm. pers.) et de Carmyerus sp.

Aucun cas de trématodose n'a été enregistré sur les 150 analyses coprologiques de moutons et de chèvres (tableau II).

Les mares alimentées par les marigots du Djeuss étaient à sec à partir de décembre. En 1993 il y a eu des lâchages d'eau depuis le barrage de Diama, ce qui a permis de réalimenter ces mares, créant des conditions favorables pour les mollusques. Ainsi l'eau et les maigres pâturages autour de ces mares ont retenu les animaux, particulièrement les bovins, dans cette zone.

Deux mares ont été prospectées dans la zone de Takhembeut et seuls Bulinus truncatus (35 spécimens) et Lymnaea natalensis (15 spécimens) ont été récoltés. Les densités étaient faibles et aucune infestation n'a été révélée.

\section{Situation dans les foyers du lac de Guiers}

\section{- Foyer de Senda}

Senda est une localité située entre Mbane et Temeye. En octobre 1991, un premier sondage coprologique réalisé sur 80 petits ruminants et 100 bovins a permis aux auteurs de constater l'existence de trématodoses dans cette zone. Les prévalences enregistrées étaient les suivantes :

- 5 p. 100 de fasciolose et 20 p. 100 de paramphistomose chez les bovins ;

- 20 p. 100 de fasciolose et 15 p. 100 de paramphistomose chez les petits ruminants (tableau II).

Cette situation épidémiologique a évolué et en janvier 1992 de nombreux cas de mortalité ont été signalés chez les petits ruminants (100) au niveau de cinq troupeaux.

En mars 1992, une enquête a été réalisée à Senda sur un total de 200 petits ruminants et 150 bovins. Les analyses coprologiques ont donné les résultats suivants :

- 30 p. 100 de fasciolose et 40 p. 100 de paramphistomose chez les bovins ;

- 55 p. 100 de fasciolose et 25 p. 100 de paramphistomose chez les petits ruminants (tableau II).

\section{- Foyers de Temeye et de Thiago}

En octobre et en novembre 1992, de nombreux cas de mortalité ont été signalés parmi des troupeaux de bovins et de petits ruminants à Thiago et à Temeye dans la zone du lac de Guiers.

Suite à cette situation, une enquête a été effectuée en janvier 1993 et a permis de dénombrer 300 bovins morts au niveau de deux troupeaux comprenant un total de 850 animaux, soit 35 p. 100 .

Seuls les animaux qui utilisaient les parcours jouxtant le lac de Guiers ont été atteints. Ceux qui vivaient hors de cette zone n'ont pas été touchés.

Les enquêtes coprologiques réalisées au niveau des différents troupeaux et les autopsies d'animaux malades ont révélé l'importance de la fasciolose à Fasciola gigantica comme cause principale de la morbidité et de la mortalité observées chez les animaux.
A Temeye, sur les 200 bovins observés, les prévalences suivantes ont été enregistrées : fasciolose 86 p. 100, paramphistomose 29 p. 100 et schistosomose 6 p. 100. Un polyparasitisme chronique a été observé avec des cas d'association parasitaire comme F. gigantica et Paramphistomum sp. (26 p. 100). Concernant les petits ruminants, sur les 100 ovins observés, une prévalence de 28 p. 100 pour la fasciolose et de 15 p. 100 pour la paramphistomose a été enregistrée (tableau II).

A Thiago, les analyses coprologiques sur 150 bovins ont révélé les prévalences suivantes : fasciolose 50 p. 100, paramphistomose 33 p. 100 et schistosomose 11 p. 100 (tableau II). Plusieurs types d'association parasitaire ont été observés chez ces animaux : F. gigantica et Paramphistomum sp. (28 p. 100), F. gigantica et Schistosoma bovis (11 p. 100), et F. gigantica, Paramphistomum sp. et Schistosoma bovis (6 p. 100). Les animaux étaient fortement infestés.

En février et en avril 1993, des prospections malacologiques au niveau des différents points d'abreuvement ont permis de récolter de nombreux mollusques hôtes intermédiaires : Biomphalaria pfeifferi, Lymnaea natalensis, Bulinus truncatus et B. forskalii. Les Lymnaea et les Biomphalaria étaient majoritaires avec des densités respectives de 146 et 86 . Dans la zone du lac, à Temeye et à Thiago, les Lymnaea étaient infestées par Fasciola gigantica avec un taux de 15 p. 100.

Entre Temeye et Thiago il y avait des zones où le lac n'était pas accessible au bétail (terres réservées aux cultures). Ainsi des mares artificielles ont été aménagées à certains endroits pour l'abreuvement du bétail et étaient alimentées en eau par pompage à partir du lac. Ces mares étaient indemnes de mollusques et les animaux qui les fréquentaient n'étaient pas atteints par la fasciolose.

\section{DISCUSSION}

Depuis les années 1970, des conditions écologiques défavorables (période de sécheresse qui a duré une dizaine d'années) avaient réduit très fortement les populations de mollusques, ayant ainsi entraîné la disparition progressive de la fasciolose devenue très rare dans le bassin du fleuve Sénégal, ainsi qu'une baisse sensible de la schistosomose et des paramphistomoses. De 1974 à 1984, les prévalences enregistrées aux abattoirs de Saint-Louis, Richard-Toll et Dagana ont confirmé la diminution de ces trématodoses : fasciolose 11 p. 100, schistosomose 15 p. 100 et paramphistomose 30 p. $100(16,17)$. Cette forte diminution des prévalences des trématodoses, particulièrement la fasciolose, de 1971 à 1985 était l'une des conséquences des dix années consécutives de sécheresse.

Actuellement, les modifications du milieu induites par la mise en service des barrages ont créé des conditions favorables à la prolifération de mollusques hôtes intermédiaires de trématodes (7). Ainsi, la période de 1988 à 1994 a été marquée par un développement et une extension des trématodoses animales (fasciolose, schistosomose, paramphistomose) avec une très forte progression des prévalences des maladies (figure 2) et la multiplication de nouveaux foyers de fasciolose et de schistosomose (figure 3). Les bovins étaient les plus atteints, mais il faut cependant signaler dans cette zone la prévalence de ces maladies chez les petits ruminants avec des taux d'infestation souvent très élevés (15).

Les trématodoses principales étaient la fasciolose à Fasciola gigantica, les paramphistomoses et les schistosomoses. Schistosoma bovis était plus fréquent que $S$. curassoni (6) en raison de la plus 
vaste distribution de son hôte intermédiaire $B$. truncatus. Cependant, il faut enregistrer la nouvelle colonisation du marigot Lampsar par B. globosus $(19,20)$, hôte potentiel de S. curassoni. En effet, après la mise en service du barrage de Diama, une plus grande distribution des bulins, avec souvent de fortes densités, a été constatée dans le delta et le lac de Guiers (7).

Cette évolution des trématodoses était bien plus nette au niveau du lac de Guiers, particulièrement avec la fasciolose et les paramphistomoses. Cependant il faut souligner qu'au niveau des deux zones (delta et lac de Guiers), les prévalences étaient plus élevées que celles observées en 1985, particulièrement à partir de 1990-91 (figure 2). C'est effectivement à cette même période que sont apparus les nouveaux foyers dans les deux zones.

Au niveau des nouveaux foyers, les prévalences de la fasciolose et des paramphistomoses observées chez les animaux du lac de Guiers étaient plus élevées que celles enregistrées au niveau du delta (figure 3).

L'extension de ces trématodoses s'est produite suivant un axe nord-sud/sud-ouest au niveau du lac de Guiers (de Richard-Toll à Keur Momar Sarr) et du delta (de Ross-Béthio à Saint-Louis).

Les taux actuels des trématodoses étaient équivalents sinon supérieurs à ceux révélés dans le bassin du fleuve Sénégal avant les années de sécheresse.

Cette situation d'évolution et d'extension des trématodoses animales est intimement liée à la prolifération des mollusques pulmonés observée dans le delta et le lac de Guiers après la mise en service des barrages de Diama et de Manantali. Cette pullulation d'hôtes intermédiaires de trématodes constitue une des premières conséquences des changements écologiques intervenus dans le bassin (7).

Après la construction du barrage de Diama et le retour d'une pluviométrie proche de la normale, cette situation épidémiologique évolutive a été favorisée par certains facteurs écologiques et épidémiologiques tels que :

- la présence permanente d'eau douce au niveau du fleuve et des marigots (barrages de Diama et de Manantali), et la régularisation du niveau d'eau dans le lac de Guiers ;

- le développement de l'irrigation et la multiplication des aménagements hydro-agricoles ;

- la pullulation des mollusques vecteurs et la colonisation de nouveaux points d'eau ;

- la forte concentration du bétail autour des points d'eau ;

- la forte crue du fleuve Sénégal de l'année 1994 qui a favorisé l'extension des mollusques vers d'autres zones transportant ainsi les métacercaires et la distomatose.

De tous ces facteurs, le plus déterminant est la prolifération des mollusques (7), en particulier les pulmonés, hôtes intermédiaires de trématodes (5).

Le fait le plus remarquable au niveau de ces foyers est le polyparasitisme chronique, dominé par des trématodes, la pathologie dominante sévissant chez le bétail dans la zone du delta et du lac de Guiers (10).

L'incidence de ces trématodoses sur la santé et les productions animales est considérable, allant d'une simple perte de poids à la mort des animaux les plus atteints, et entraîne des pertes économiques importantes.

\section{- CONCLUSION}

Cette nouvelle situation épidémiologique découle des bouleversements écologiques survenus ces dernières années au niveau du bassin du fleuve Sénégal et du lac de Guiers. On assiste à un développement et à une extension des foyers de fasciolose et de schistosomose animales ainsi que de ceux de bilharzioses humaines. Cette situation est d'autant plus préoccupante pour le Sénégal qu'elle peut concerner des projets en cours, tels la remise en eau des vallées fossiles et le creusement du canal du Cayor, canal à ciel ouvert devant relier le lac de Guiers à Dakar, à 200 km plus au sud.

Une surveillance épidémiologique et malacologique constante ainsi qu'un plan d'urgence de lutte contre les trématodes et les mollusques vecteurs sont nécessaires dans tout le bassin du fleuve Sénégal où les conditions écologiques sont très favorables à l'extension des trématodoses.

\section{BIBLIO GRAPHIE}

1. ALBARET J.L., BAYSSADE-DUFOUR CH., DIAW O.T., VASSILIADES G., GRUNER L., 1980. Données complémentaires sur les organites argyrophiles superficiels du miracidium et de la cercaire de Fasciola gigantica (Cobbold, 1855) (Trematoda : Fasciolidae) et sur l'épidémiologie de ce parasite. Ann. Parasitol. (Paris), 55 : 541-552.

2. ALBARET J.L., BAYSSADE-DUFOUR CH., DIAW O.T., VASSILIADES G., SEY O., GRUNER L., 1981. Description des organites argyrophiles superficiels du miracidium et de la cercaire de Paramphistomum phillerouxi Dinnik, 1961. Ann. Parasitol. (Paris), 56 : 127-154.

3. BROWN D., 1994. Fresh water snails of Africa and their medical importance, 2nd ed. London, UK, Taylor and Francis Ltd, 609 p.

4. COGELS F.X., THIAM A., GAC J.I., 1993. Premiers effets des barrages du fleuve Sénégal sur le lac de Guiers. Revue Hydrobiol. trop., 26 : 105-117.

5. DIAW O.T., PICOT H., ALBARET I.L., BAYSSADE-DUFOUR CH., VASSILIADES G., 1986. Rôle épidémiologique des mollusques du genre Bulinus dans la transmission des schistosomiases humaines et animales au Sénégal. Revue méd. Côte d'Ivoire, 20e année, (75) : 183.

6. DIAW O.T., VASSILIADES G., 1987. Epidémiologie des schistosomoses du bétail au Sénégal. Revue Elev. Méd. vét. Pays trop., $40: 265-274$

7. DIAW O.T., VASSILIADES G., SEYE M., SARR Y., 1990. Prolifération de mollusques et incidence sur les trématodoses dans la région du delta et du lac de Guiers après la construction du barrage de Diama sur le fleuve Sénégal. Revue Elev. Méd. vét. Pays trop., 43 : 499-502.

8. EUZEBY J., 1958. Diagnostic expérimental des helminthoses animales. Travaux pratiques d'helminthologie vétérinaire. Paris, France, Vigot frères.

9. FRANDSEN F., CHRISTEN SEN N.O ., 1984. An introductory guide to identification of cercariae from African freshwater snails with reference to cercariae of trematode species of medical and veterinary importance. Acta tropica, 41: 181-202.

10. KABORET Y.Y., THIONGANE Y., SAWADOGO G., AKAKPO A.J., 1993. Etude anatomo-clinique d'un cas de polyparasitisme à Fasciola gigantica et à Schistosoma bovis chez un zébu Peulh au Sénégal. Revue Méd. vét., 144 : 759-765.

11. MALEK E.A., CHAINE J.P., 1989. Effect on the developments in the Senegal River basin on the prevalence and spread of schistosomiasis. In: M.W. Service Ed., Demography and vector borne diseases. Boca Raton, FL, USA, CRC Press, p. 181-192.

12. MANDAHL BARTH G., 1973. A field guide of African fresh water snails: 1. West African species (Senegal - Nigeria). Charlottenlund, Denmark, WHO Snail Identification Centre, Danish Bilharziasis Laboratory, $29 \mathrm{p}$. 
13. RICHARD J., 1971. La chétotaxie des cercaires. Valeur systématique et phylétique. Mém. Mus. natl Hist. nat., Paris, Ser. A Zool., 67 : 1-179.

14. TALLA I., KONGS A., VERLE P., BELOT J., SARR S., COLL A.M., 1990. O utbreak of intestinal schistosomiasis in the Senegal River basin. Ann. Soc. Belge Méd. trop., 70: 173-180.

15. TOURAND J.F., 1994. L'élevage dans la révolution agricole au Waalo. Ruptures et continuités. Thèse doct. Etat, U niversité Paris XII, Créteil, France, $416 \mathrm{p}$.

16. VASSILIADES G., 1974. Les affections parasitaires à helminthes chez les bovins domestiques de la région du fleuve Sénégal. Bull. Epizoot. Afr., 22 : 74-78.

17. VASSILIADES G., 1978. Les affections parasitaires dues à des helminthes chez les bovins du Sénégal. Revue Elev. M éd. vét. Pays trop., $31: 157-163$

\section{Summary}

Diaw O.T., Vassiliades G., Thiongane Y., Seye M., Sarr Y., D iouf A. Spread of cattle trematodoses after dam building in the Senegal River basin

After Diama dam became operational in 1985-1986 and many hydro-agricultural points were set up, an increase in animal trematodoses was observed in the Senegal River basin. It concerned in particular Fasciola gigantica, Schistosoma bovis, S. curassoni and Paramphistomum sp. The cattle trematodosis epidemiology showed itself in the disease prevalence increase in existing foci (Richard-Toll, RossBéthio, M bane et Keur Momar Sarr). Infestation rates in cattle increased from 11 to $27 \%, 20$ to $30 \%$ and 15 to $27 \%$ for fasciolosis, paramphistomosis and schistosomosis, respectively. In small ruminants, who had seemed spared, 2-62\% fasciolosis prevalence rates were recorded, whereas they were 25-30\% for paramphistomosis. In a parallel manner, new trematodosis foci appeared starting in 1989-1990: 1) at the delta level in Tilène, Pont Gendarme et Takhembeut with 3-20, 4-20 and 5-36\% prevalence rates for fasciolosis, schistosomosis and paramphistomosis, respectively; and 2) at the Guiers Lake level in Temeye, Thiago et Senda with 5-86, 5-11 and 5-33\% prevalence rates for fasciolosis, schistosomosis and paramphistomosis, respectively. In these new foci, $2-55$ and $5-25 \%$ prevalence rates were recorded in small ruminants for fasciolosis and paramphistomosis, respectively. Schistosomosis was not as frequent with $2-4 \%$ prevalence rates. This new trematodosis epidemiology in the Senegal River basin starting in 1988-1989 was chiefly remarkable by very high infection rates and parasite burdens, and by a polyparasitism which combined Fasciola gigantica, Schistosoma bovis, S. curassoni and Paramphistomum sp.

Key words: Cattle - Goat - Sheep - Fasciola gigantica Schistosoma bovis - Paramphistomum sp. - Epidemiology Dam - Delta - Lake - Senegal.
18. VERCRUYSSE J., SCANDEVYL P., 1984. Parasitological and pathological observations in schistosomiasis in sheep in Senegal. J. Helminth., 58: 219- 220.

19. VERCRUYSSE J., SOUTHGATE V.R., ROLLINSON D., 1985. Epidemiology of human and animal schistosomiasis in the Senegal River basin. Acta tropica, 42: 249-259.

20. VERLE P., STELMA F., DESREU MAUX P., DIENG A., DIAW O.T., KONGS A., NIANG M., SOW S., TALLA I., STURROCK R.F., GRYSEELS B., CAPRON A., 1994. Preliminary study of urinary schistosomiasis in a village in the delta of the Senegal River basin, Senegal. Trans. R. Soc. trop. M ed. Hyg., 88: 401-405.

Reçu le 14.2.97, accepté le 17.8.98

\section{Resumen}

Diaw O.T., Vassiliades G., Thiongane Y., Seye M., Sarr Y., Diouf A. Extensión de las infecciones por tremátodos en el ganado, después de la construcción de represas en la cuenca del río Senegal

Después del inicio del funcionamiento de la represa de Diama (1985-1986) y de la multiplicación de las obras hidroagrícolas, se ha constatado, a nivel de la cuenca del río Senegal, un desarrollo de las infecciones animales por tremátodos, particularmente la fasciolosis por Fasciola gigantica, las esquistosomosis por Schistosoma bovis y por $\mathrm{S}$. curassoni y la paranfistomosis por Paramphistomum sp. Este cuadro epidemiológico de los tremátodos en el ganado se ha manifestado por un aumento de las prevalencias a nivel de Ios antiguos focos (Richard-Toll, Ross-Bethio, M bane y Keur Momar Sarr). Las tasas de infestación en los bovinos pasaron de 11 a $27 \%$, de 20 a $30 \%$ y de 15 a $27 \%$ para la fasciolosis, la paranfistomosis y la esquistosomosis respectivamente. En los pequeños rumiantes, que parecían ilesos, se registraron prevalencias de 2 a $62 \%$ para la faciolosis, mientras que la paranfistomosis, más frecuente, tuvo una tasa de 25 a 30\%. En forma paralela, a partir de 1989-1990, aparecieron nuevos focos de tremátodos: por un lado a nivel del delta en Tilene, Puerto Gendarme y Takhembeut, con prevalencias de 3 a $20 \%, 4$ a $20 \%$ y de 5 a $36 \%$ respectivamente para la fasciolosis, esquistosomosis y paranfistomosis. Por otro lado, a nivel del lago de Guiers, en Temeye, Thiago y Senda, con prevalencias de 5 a $86 \%$, de 5 a $11 \%$ y de 5 a $33 \%$ respectivamente para la fasciolosis, esquistosomosis y paranfistomosis. En estos nuevos focos, a nivel de los pequeños rumiantes, se registraron prevalencias de 2 a $55 \%$ para la fasciolosis y de 5 a $25 \%$ para la paranfistomosis. La esquistosomosis fue menos frecuente, con prevalencias de 2 a $4 \%$. Esta nueva situación epidemiológica de los tremátodos (fasciolosis, esquistosomosis y paranfistomosis) en la cuenca del río Senegal, a partir de 1989 1990, es importante principalmente por las altas prevalencias de estas afecciones, las fuertes cargas parasitarias y por un poliparasitismo asociado a fasciolas, esquistosomas y paranfistomos.

Palabras clave: Ganado bovino - Caprino - Ovino - Fasciola gigantica - Schistosoma bovis - Paramphistomum sp. Epidemiología - Represa - Delta - Lago - Senegal. 